\title{
ORT_18 - Cell penetrating peptides functionalization in polymeric nanoparticles containing antiviral as a strategy for the Neurocovid treatment
}

Melissa Chamon Alves Premazzi ${ }^{1 *}$; Kaique Alves Brayner Pereira ${ }^{1}$; Vinícius de Lima Gonçalves ${ }^{1}$; Luãnna Elisa Liebscher Vidal ${ }^{1}$; Rayane de Oliveira Guerra ${ }^{1}$; Luciana da Silva Madeira ${ }^{1}$; Izabella Sodré Buty da Silva $^{1}$; Patricia Barbosa Jurgilas ${ }^{1}$; Renata Chagas Bastos ${ }^{1}$.

${ }^{1}$ Fiocruz/Bio-Manguinhos.

Introduction: Neurological manifestations have been observed in patients affected by coronavirus 19 disease since the emergence of the pandemic. Although questions remain about the frequency and severity of this condition and regarding which factors may predispose to the neurological condition, therapeutic strategies to inhibit this action of the coronavirus are necessary. Polymeric nanoparticles (PNPs) have been shown to be interesting carriers, presenting adequate characteristics of stability, biodegradability and low toxicity. The functionalization of PNPs with cell penetrate peptides (CPPs) is a targeting strategy, that enables to translocate by the plasma membrane and facilitates the antiviral release. CPPs are small, highly cationic peptide chains acting as drug delivery agents to target cells. In this work, the optimal condition of functionalization of PNPs with CPP will be evaluated, which will potentially lead PNPs containing the antiviral through the central nervous system for the neurocovid treatment.

Objective: Functionalization and characterization of PNPs with CPPs obtained by enzimatic hydrolysis, purification and activation.

Methodology: CPPs were obtained by enzymatic hydrolysis in buffered medium ( $\mathrm{pH}$ 6.8). The nonhydrolyzed peptide and remained enzyme were removed by ultrafiltration. Hydrolysis efficiency was evaluated by fluorescence spectroscopy using extrinsic Bis-ANS probe. CPPs were purified by affinity chromatography with ultraviolet (UV) detection and the molecular mass was estimated by SEC. CPPs were activated by reaction with the succinimidyl-3-(2-pyridyldithio)-propionate reagent and subsequent reduction with excess dithiothreitol. Its concentration was determined by visible absorption spectrophotometry after colorimetric reaction with Ellman's reagent. The PNPs were functionalized by the direct reaction with CPPs, under agitation for 16-20h. The precipitate was resuspended in water. Functionalization efficiency was assessed by determining the mean diameter and zeta potential obtained by the dynamic light scattering technique and comparing of the spectroscopy data in the infrared region of funcionalizated and non-functionalized PNPs.

Results: After determining the optimal hydrolysis condition, obtained CPPs were evaluated by the emission intensity decrease in the fluorescence spectrum as a function of the incubation time with the enzyme, The results confirmed the efficiency of hydrolysis. Chromatographic profile of the purification was similar to that described in the literature and allowed to select one of the five peaks observed. After activation, the concentration of CPPs were estimated in $0.28 \mathrm{mg} / \mathrm{mL}$ with an average molecular mass of 1380Da. The efficiency of functionalization of antiviral PNPs with the activated CPPs was evaluated by the mean diameter, zeta potential and IR spectra for non-functionalized PNPs compared to those obtained after functionalization. The results suggests that reaction conditions employed were efficient to obtain PNPs linked to CPPs.

Conclusion: The reaction conditions used to obtain the antiviral PNPs suggest the efficiency of functionalization with CPPs. The functionalized PNPs can be tested by in vitro assays to neurocovid treatment.

Keywords: polymeric nanoparticles; funcionalization; neurocovid 